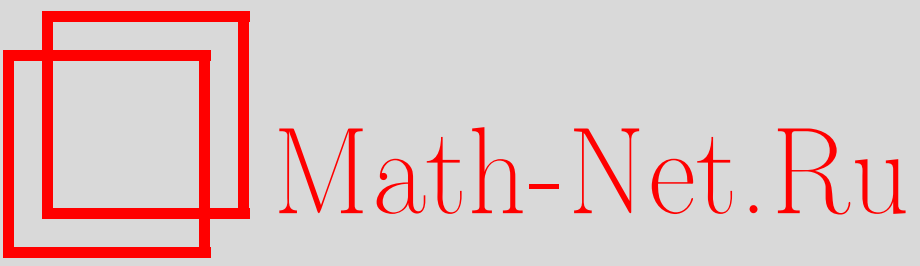

Е. В. Ферапонтов, Преобразования Лапласа систем гидродинамического типа в инвариантах Римана, ТМФ, 1997, том 110, номер 1, 86-97

DOI: https://doi.org/10.4213/tmf954

Использование Общероссийского математического портала Math-Net.Ru подразумевает, что вы прочитали и согласны с пользовательским соглашением

http://www.mathnet.ru/rus/agreement

Параметры загрузки:

IP: 52.23 .180 .231

26 апреля 2023 г., 07:18:29 


\section{ПРЕОБРАЗОВАНИЯ ЛАПЛАСА СИСТЕМ ГИДРОДИНАМИЧЕСКОГО ТИПА В ИНВАРИАНТАХ РИМАНА}

Плотности законов сохранения системы гидродинамического типа в инвариантах Римана описываются системой линейных дифференциальных уравнений второго порядка. Для линейных систем такого вида Дарбу ввел преобразования Лапласа, обобщающие классические преобразования скалярного уравнения второго порядка. В работе показано, что преобразования Лапласа "поднимаются" до преобразований соответствующих систем гидродинамического типа. Изучаются конечные семейства систем гидродинамического типа, замкнутые относительно всей совокупности преобразований Лапласа. Для систем порядка $3 \times 3$ в инвариантах Римана дано описание замкнутых четверок. Они оказались связанными со специальной квадратичной редукцией $(2+1)$-мерной системы трех волн.

\section{1. ФОРМУЛИРОВКА РЕЗУЛЬТАТА}

Рассмотрим двухкомпонентную систему гидродинамического типа в инвариантах Римана

$$
\begin{aligned}
& R_{t}^{1}=\lambda^{1}(R) R_{x}^{1} \\
& R_{t}^{2}=\lambda^{2}(R) R_{x}^{2}
\end{aligned}
$$

Плотности $h$ законов сохранения системы (1) удовлетворяют линейному уравнению второго порядка

$$
\partial_{1} \partial_{2} h=a \partial_{1} h+b \partial_{2} h
$$

где

$$
a=\frac{\partial_{2} \lambda^{1}}{\lambda^{2}-\lambda^{1}}, \quad b=\frac{\partial_{1} \lambda^{2}}{\lambda^{1}-\lambda^{2}},
$$

$\partial_{i}=\frac{\partial}{\partial R^{i}}$. Применяя к уравнению (2) преобразование Лапласа, т.е. переходя к новой функции

$$
H=h-\frac{\partial_{1} h}{b},
$$

мы получим относительно $H$ уравнение того же вида

$$
\partial_{1} \partial_{2} H=A \partial_{1} H+B \partial_{2} H
$$

${ }^{*}$ Институт математического моделирования РАН, Центр нелинейных исследований ИТФ им. Л. Д. Ландау 
где

$$
A=a-\frac{\partial_{2} b}{b}, \quad B=b+\partial_{1} \ln A
$$

(обратное преобразование $H=h-\frac{\partial_{2} h}{a}$ рассматривается аналогично). Оказывается, что преобразование Лапласа можно "поднять" до преобразования соответствуюших систем гидродинамического типа. Введем в рассмотрение систему

$$
\begin{aligned}
& R_{t}^{1}=\Lambda^{1}(R) R_{x}^{1}, \\
& R_{t}^{2}=\Lambda^{2}(R) R_{x}^{2},
\end{aligned}
$$

где новые характеристические скорости $\Lambda^{1}, \Lambda^{2}$ связаны с $\lambda^{1}, \lambda^{2}$ формулами

$$
\begin{aligned}
\Lambda^{1} & =\lambda^{2}, \\
\Lambda^{2}=\lambda^{2}-\frac{b \partial_{2} \lambda^{2}}{\partial_{2} b-a b} & =\lambda^{2}-\frac{\partial_{2} \lambda^{2}}{\frac{\partial_{1} \partial_{2} \lambda^{2}}{\partial_{1} \lambda^{2}}+\frac{\partial_{2} \lambda^{2}}{\lambda^{1}-\lambda^{2}}} .
\end{aligned}
$$

ТЕОРема 1. 1. Плотности законов сохранения Н системъ (5) удовлетворяют уравнению (3) и вычисляются по формуле

$$
H=h-\frac{\partial_{1} h}{b} .
$$

2. Характеристические скорости $W^{1}, W^{2}$ коммутирующих потоков системы (5) вычисляются по формулам

$$
\begin{aligned}
& W^{1}=w^{2}, \\
& W^{2}=w^{2}-\frac{b \partial_{2} w^{2}}{\partial_{2} b-a b},
\end{aligned}
$$

где $w^{1}, w^{2}$ - характеристические скорости коммутирующих потоков исходной системь (1), т.е. решения линейной системь (см. [1])

$$
\frac{\partial_{2} w^{1}}{w^{2}-w^{1}}=a, \quad \frac{\partial_{1} w^{2}}{w^{1}-w^{2}}=b .
$$

Доказательство теоремы состоит в непосредственной проверке соотношений

$$
A=\frac{\partial_{2} \Lambda^{1}}{\Lambda^{2}-\Lambda^{1}}, \quad B=\frac{\partial_{1} \Lambda^{2}}{\Lambda^{1}-\Lambda^{2}}
$$

а также

$$
\frac{\partial_{2} W^{1}}{W^{2}-W^{1}}=A, \quad \frac{\partial_{1} W^{2}}{W^{1}-W^{2}}=B .
$$

Систему (5) естественно назвать преобразованием Лапласа системы (1). Преобразование Лапласа очевидным образом сохраняет "интегрируемость" : зная плотности законов сохранения и коммутируюшие потоки исходной системы (1), мы автоматически пересчитываем их в плотности законов сохранения и коммутируюшие потоки системы (5) по 
формулам, приведенным в теореме. В частности, решения исходной системы, задаваемые формулой годографа (см. [1])

$$
w^{1}=x+\lambda^{1} t, \quad w^{2}=x+\lambda^{2} t
$$

переходят в решения

$$
W^{1}=x+\Lambda^{1} t, \quad W^{2}=x+\Lambda^{2} t
$$

системы (5).

Обратному преобразованию Лапласа $H=h-\frac{\partial_{2} h}{a}$ отвечает замена индексов 1 и 2 в формулах (6). Дальнейшие свойства преобразований Лапласа в двухкомпонентном случае обсуждаются в п. 2 .

Преобразования Лапласа обобшаются на системы из $n$ уравнений в инвариантах Римана

$$
R_{t}^{i}=\lambda^{i}(R) R_{x}^{i}
$$

Для любой пары индексов $i \neq j$ определено преобразование $S_{i j}$, переводяшее систему (7) в новую систему

$$
R_{t}^{i}=\Lambda^{i}(R) R_{x}^{i}
$$

характеристические скорости которой вычисляются по формулам

$$
\begin{gathered}
\Lambda^{i}=\lambda^{j}, \\
\Lambda^{j}=\lambda^{j}-\frac{a_{j i} \partial_{j} \lambda^{j}}{\partial_{j} a_{j i}-a_{i j} a_{j i}}, \\
\Lambda^{k}=\frac{\lambda^{k} a_{j i}-\lambda^{j} a_{k i}}{a_{j i}-a_{k i}}, \quad k \neq i, j,
\end{gathered}
$$

где $a_{j i}=\frac{\partial_{i} \lambda^{j}}{\lambda^{i}-\lambda^{j}}$.

Теорема 2. 1. Плотности законов сохранения $H$ преобразованной системы связаны с плотностями $h$ исходной системы формулой

$$
H=h-\frac{\partial_{i} h}{a_{j i}}
$$

2. Характеристические скорости $W^{i}$ коммутирующих потоков преобразованной системы вычисляются по формулам

$$
\begin{gathered}
W^{i}=w^{j}, \\
W^{j}=w^{j}-\frac{a_{j i} \partial_{j} w^{j}}{\partial_{j} a_{j i}-a_{i j} a_{j i}}, \\
W^{k}=\frac{w^{k} a_{j i}-w^{j} a_{k i}}{a_{j i}-a_{k i}}, \quad k \neq i, j,
\end{gathered}
$$


где $w^{i}$ - характеристические скорости коммутирующих потоков исходной системы, т.е. решения линейной системы (см. [1])

$$
\frac{\partial_{j} w^{i}}{w^{j}-w^{i}}=a_{i j}, \quad i \neq j
$$

Напомним, что плотности $h$ законов сохранения исходной системы (7) удовлетворяют переопределенной системе линейных уравнений второго порядка

$$
\partial_{i} \partial_{j} h=a_{i j} \partial_{i} h+a_{j i} \partial_{j} h, \quad i \neq j
$$

условиями совместности которой являются соотношения

$$
\partial_{k} a_{i j}=a_{i k} a_{k j}+a_{i j} a_{j k}-a_{i j} a_{i k}, \quad i \neq j \neq k \neq i .
$$

Формула (9) задает преобразование Лапласа переопределенной системы (10) (см. [2, с. 274]). В случае $n=2$ имеется фактически единственное преобразование $S_{12}$, т.к. $S_{12} \circ S_{21}=$ id.

Дальнейшие свойства преобразований $S_{i j}$ обсуждаются в п. 3.

\section{2. ПРЕОБРАЗОВАНИЯ ЛАПЛАСА ДВУХКОМПОНЕНТНЫХ СИСТЕМ}

Итерируя преобразование Лапласа, мы согласно формулам (6) получим бесконечный ряд систем, характеристические скорости которых являются последовательными решениями интегрируемой цепочки

$$
\frac{\partial_{1} \partial_{2} \lambda^{n}}{\partial_{1} \lambda^{n} \partial_{2} \lambda^{n}}=\frac{1}{\lambda^{n}-\lambda^{n-1}}+\frac{1}{\lambda^{n}-\lambda^{n+1}}
$$

Преобразованием Лапласа системы с характеристическими скоростями $\left(\lambda^{n-1}, \lambda^{n}\right)$ является система с характеристическими скоростями $\left(\lambda^{n}, \lambda^{n+1}\right)$, полученная сдвигом по цепочке на один шаг. В терминах коэффициентов

$$
a^{n}=\frac{\partial_{2} \lambda^{n}}{\lambda^{n+1}-\lambda^{n}}, \quad b^{n}=\frac{\partial_{1} \lambda^{n}}{\lambda^{n-1}-\lambda^{n}}
$$

цепочка (11) принимает вид

$$
\partial_{1} a^{n}=a^{n}\left(b^{n+1}-b^{n}\right), \quad \partial_{2} b^{n}=b^{n}\left(a^{n-1}-a^{n}\right)
$$

и после замены (ср. с [3])

$$
q^{n}=\ln a^{n} b^{n}=\ln \frac{\partial_{2} \lambda^{n} \partial_{1} \lambda^{n}}{\left(\lambda^{n+1}-\lambda^{n}\right)\left(\lambda^{n-1}-\lambda^{n}\right)}
$$

преврашается в известную цепочку Тоды

$$
\partial_{1} \partial_{2} q^{n}=2 e^{q^{n}}-e^{q^{n+1}}-e^{q^{n-1}}
$$


возникавшую в теории преобразований Лапласа уравнений второго порядка еше в классических работах дифференциальных геометров прошлого века.

ЗАмЕчАниЕ. Цепочка (11) отличается от известной билинейной записи цепочки Тоды

$$
f^{n} \partial_{1} \partial_{2} f^{n}-\partial_{1} f^{n} \partial_{2} f^{n}=-f^{n+1} f^{n-1},
$$

получающейся из (12) подстановкой

$$
q^{n}=\ln \frac{f^{n+1} f^{n-1}}{f^{n^{2}}} .
$$

В одномерном случае цепочка (11) возникала в [4]. Как отмечалось в [5], она задает преобразование Беклунда уравнений магнетика Гейзенберга.

Ряд красивых результатов в теории преобразований Лапласа был получен при исследовании периодических цепочек, которые, как было недавно установлено в [6], имеют глубокие связи со спектральной теорией двумерного оператора Шредингера. Оказывается, что всякая периодическая цепочка преобразований Лапласа уравнений второго порядка вида (2) поднимается до периодической цепочки преобразований Лапласа соответствующих систем гидродинамического типа (для которых данные уравнения являются уравнениями на плотности законов сохранения). Это поднятие описывается периодическими редукциями цепочки (11).

ПримеР. Рассмотрим периодическую цепочку преобразований Лапласа периода 2, в которой уравнение $(2)$ с коэффициентами $(a, b)$ переходит в уравнение $(2)$ с коэффициентами $(A, B)$, а затем обратно в $(a, b)$. При этом из (4) вытекает, что мы можем без ограничения обшности положить $A=\frac{1}{a}, B=\frac{1}{b}$ т.е.

$$
(a, b) \rightarrow\left(\frac{1}{a}, \frac{1}{b}\right) \rightarrow(a, b) .
$$

В силу (4) коэффициенты $a$ и $b$ будут удовлетворять уравнениям

$$
\partial_{2} \ln b=a-\frac{1}{a}, \quad \partial_{1} \ln a=b-\frac{1}{b},
$$

которые сводятся к уравнению sh-Gordon

$$
\partial_{1} \partial_{2} \varphi=4 \operatorname{sh} \varphi
$$

на функцию $\varphi=\ln a b$. Соответствующее цепочке (13) периодическое поднятие преобразования Лапласа имеет вид

$$
\left(\lambda^{1}, \lambda^{2}\right) \rightarrow\left(\Lambda^{1}, \Lambda^{2}\right) \rightarrow\left(\lambda^{1}, \lambda^{2}\right)
$$

где, как автоматически вытекает из формул (6), $\Lambda^{1}=\lambda^{2}, \Lambda^{2}=\lambda^{1}$. При этом коэффициенты $a, b, \lambda^{1}, \lambda^{2}$ связаны уравнениями

$$
\begin{array}{ll}
\frac{\partial_{2} \lambda^{1}}{\lambda^{2}-\lambda^{1}}=a, & \frac{\partial_{1} \lambda^{2}}{\lambda^{1}-\lambda^{2}}=b, \\
\frac{\partial_{2} \lambda^{2}}{\lambda^{1}-\lambda^{2}}=\frac{1}{a}, & \frac{\partial_{1} \lambda^{1}}{\lambda^{2}-\lambda^{1}}=\frac{1}{b}
\end{array}
$$


которые являются совместными в силу (14). Несложно показать, что сами $\lambda^{1}, \lambda^{2}$ удовлетворяют двум уравнениям второго порядка

$$
\frac{\partial_{1} \partial_{2} \lambda^{1}}{\partial_{1} \lambda^{1} \partial_{2} \lambda^{1}}=\frac{2}{\lambda^{1}-\lambda^{2}}, \quad \frac{\partial_{1} \partial_{2} \lambda^{2}}{\partial_{1} \lambda^{2} \partial_{2} \lambda^{2}}=\frac{2}{\lambda^{2}-\lambda^{1}}
$$

которые представляют собой периодическую редукцию цепочки (11) периода $2: \lambda^{3}=\lambda^{1}$, $\lambda^{4}=\lambda^{2}$. Формулы (15) описьвают периодическое поднятие цепочки преобразований Лапласа периода 2. Это поднятие определено однозначно с точностью до преобразований $\lambda^{1} \rightarrow p \lambda^{1}+q, \lambda^{2} \rightarrow p \lambda^{2}+q, p, q=$ const. Заметим, что, применяя преобразования Лапласа к коммутируюшей системе с характеристическими скоростями $w^{1}, w^{2}$, которые не удовлетворяют ограничениям (15) (хотя и отвечают тем же самым $a, b$ ), мы, вообще говоря, не вернемся к исходной системе после обхода по циклу.

ЗАмЕчАниЕ. Для периодической цепочки преобразований Лапласа периода 2

$$
(a, b) \rightarrow\left(\frac{1}{a}, \frac{1}{b}\right) \rightarrow(a, b)
$$

мы можем построить непериодическое поднятие вида

$$
\left(w^{1}, w^{2}\right) \rightarrow\left(w^{2}, \mu w^{1}\right) \rightarrow\left(\mu w^{1}, \mu w^{2}\right)
$$

где $\mu=$ const. При этом $a, b, w^{1}, w^{2}$ будут связаны соотношениями

$$
\begin{aligned}
\frac{\partial_{2} w^{1}}{w^{2}-w^{1}} & =a, & \frac{\partial_{1} w^{2}}{w^{1}-w^{2}} & =b, \\
\frac{\partial_{2} w^{2}}{\mu w^{1}-w^{2}} & =\frac{1}{a}, & \frac{\mu \partial_{1} w^{1}}{w^{2}-\mu w^{1}} & =\frac{1}{b} .
\end{aligned}
$$

Соотношения (16) являются совместными и представляют собой спектральную задачу для уравнений (14), причем $\mu$ играет роль спектрального параметра. Из формул (16) вытекает, что $\left(\lambda^{1}, \lambda^{2}\right)$ - это компоненты волновой функции $\left(w^{1}, w^{2}\right)$ при $\mu=1$.

Аналогичная ситуация имеет место и в случае периодических цепочек произвольной длины $n$. А именно, для всякой периодической цепочки преобразований Лапласа уравнений второго порядка существует каноническое поднятие с тем же периодом $n$ на соответствуюшие системы гидродинамического типа (так что рассматриваемые уравнения второго порядка являются уравнениями на плотности законов сохранения этих систем). При этом над всякой периодической цепочкой преобразований Лапласа периода $n$ имеется $n$-параметрическое семейство периодических цепочек систем гидродинамического типа. Точно так же (рассматривая непериодические поднятия) можно построить и спектральные задачи, отвечаюшие периодическим цепочкам преобразований Лапласа произвольной длины $n$. 


\section{3. ПРЕОБРАЗОВАНИЯ ЛАПЛАСА $n$-КОМПОНЕНТНЫХ СИСТЕМ}

ЛЕммА. Преобразования $S_{i j}$ удовлетворяют соотношениям

$$
\begin{gathered}
S_{i j} \circ S_{j i}=\mathrm{id}, \\
S_{i j}=S_{i k} \circ S_{k j}=S_{k j} \circ S_{i k}, \quad k \neq i, j .
\end{gathered}
$$

Доказательство леммы может быть получено непосредственным вычислением. Аналогичные соотношения хорошо известны в теории преобразований Лапласа $n$-сопряженных систем координат (см., например, [2, с. 275]). Как вытекает из (17), преобразования $S_{i j}$ порождают свободную абелеву группу с $(n-1)$ образующими (в качестве образуюших могут быть выбраны, например, $\left.S_{12}, \ldots, S_{1 n}\right)^{1)}$.

На языке сопряженных систем координат свойства преобразований $S_{i j}$ изучались и в более поздних работах [7-9], авторам которых, по-видимому, были неизвестны исследования Дарбу [2]. Мы отсылаем читателя к работе [10] за дальнейшей библиографией по преобразованиям Лапласа сопряженных систем координат. На языке многообразий Картана преобразование Лапласа $n$-сопряженных систем координат изучалось в недавней работе [11]. Отметим также близкую по подходу работу [12], где методом факторизации были построены аналоги преобразований Лапласа многомерных матричных дифференциальных операторов первого порядка.

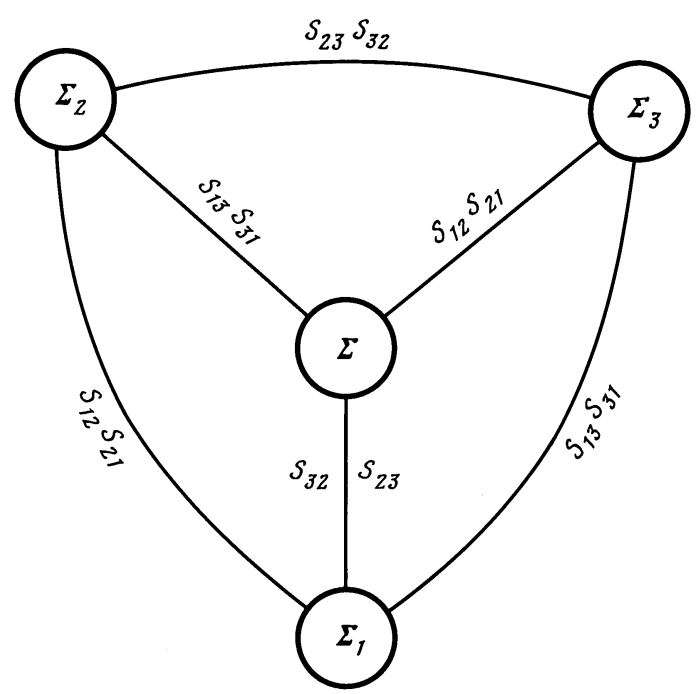

Представляет интерес дальнейшее изучение свойств преобразований $S_{i j}$, а именно: изучение конечных семейств систем гидродинамического типа, замкнутых относительно всей совокупности преобразований $S_{i j}$ (аналогов замкнутых цепочек преобразований Лапласа в случае $n=2)$. Естественно предположить, что такие системы будут обладать "повышенной" интегрируемостью;

исследование вопроса о поведении гамильтоновых структур при преобразованиях $S_{i j}$.

\footnotetext{
1) Автор благодарит участников семинара "Геометрия и математическая физика" под руководством С. П. Новикова за прояснение этого обстоятельства.
} 
В заключение дадим описание четверок систем гидродинамического типа порядка $3 \times 3$, замкнутых относительно всей совокупности преобразований Лапласа. Пусть характеристические скорости систем $\Sigma, \Sigma_{1}, \Sigma_{2}, \Sigma_{3}$ равны соответственно $\left(\lambda^{1}, \lambda^{2}, \lambda^{3}\right)$, $\left(\lambda^{4}, \lambda^{3}, \lambda^{2}\right),\left(\lambda^{3}, \lambda^{4}, \lambda^{1}\right),\left(\lambda^{2}, \lambda^{1}, \lambda^{4}\right)$ (см. рисунок).

Линии с пометками, соединяющие пары систем на рисунке, означают, что, например, система $\Sigma_{3}$ получается из $\Sigma$ под действием преобразований Лапласа $S_{12}$ и $S_{21}$ (и обратно, $\Sigma$ получается из $\Sigma_{3}$ под действием $S_{12}$ и $\left.S_{21}\right)$. Расстановка пометок согласована с тождествами (17). Поскольку в нашей конструкции $S_{i j}^{2}=\mathrm{id}$ для любой пары индексов $i, j$, рассматриваемая четверка систем является аналогом обсуждавшихся ранее в случае $n=2$ периодических цепочек преобразований Лапласа периода 2. Соотношения (8) приводят к сложной переопределенной системе уравнений на характеристические скорости $\lambda^{1}-\lambda^{4}$ :

$$
\begin{array}{rlrl}
\frac{\partial_{1} \partial_{2} \lambda^{1}}{\partial_{1} \lambda^{1} \partial_{2} \lambda^{1}} & =\frac{2}{\lambda^{1}-\lambda^{2}}, & \frac{\partial_{1} \partial_{2} \lambda^{2}}{\partial_{1} \lambda^{2} \partial_{2} \lambda^{2}} & =\frac{2}{\lambda^{2}-\lambda^{1}}, \\
\frac{\partial_{1} \partial_{3} \lambda^{1}}{\partial_{1} \lambda^{1} \partial_{3} \lambda^{1}}=\frac{2}{\lambda^{1}-\lambda^{3}}, & \frac{\partial_{1} \partial_{3} \lambda^{3}}{\partial_{1} \lambda^{3} \partial_{3} \lambda^{3}}=\frac{2}{\lambda^{3}-\lambda^{1}}, \\
\frac{\partial_{2} \partial_{3} \lambda^{2}}{\partial_{2} \lambda^{2} \partial_{3} \lambda^{2}}=\frac{2}{\lambda^{2}-\lambda^{3}}, & \frac{\partial_{2} \partial_{3} \lambda^{3}}{\partial_{2} \lambda^{3} \partial_{3} \lambda^{3}}=\frac{2}{\lambda^{3}-\lambda^{2}}, \\
\frac{\partial_{1} \partial_{2} \lambda^{3}}{\partial_{1} \lambda^{3} \partial_{2} \lambda^{3}}=\frac{2}{\lambda^{3}-\lambda^{4}}, & \frac{\partial_{1} \partial_{2} \lambda^{4}}{\partial_{1} \lambda^{4} \partial_{2} \lambda^{4}}=\frac{2}{\lambda^{4}-\lambda^{3}}, \\
\frac{\partial_{1} \partial_{3} \lambda^{2}}{\partial_{1} \lambda^{2} \partial_{3} \lambda^{2}}=\frac{2}{\lambda^{2}-\lambda^{4}}, & \frac{\partial_{1} \partial_{3} \lambda^{4}}{\partial_{1} \lambda^{4} \partial_{3} \lambda^{4}}=\frac{2}{\lambda^{4}-\lambda^{2}}, \\
\frac{\partial_{2} \partial_{3} \lambda^{1}}{\partial_{2} \lambda^{1} \partial_{3} \lambda^{1}}=\frac{2}{\lambda^{1}-\lambda^{4}}, & \frac{\partial_{2} \partial_{3} \lambda^{4}}{\partial_{2} \lambda^{4} \partial_{3} \lambda^{4}}=\frac{2}{\lambda^{4}-\lambda^{1}},
\end{array}
$$

а также

$$
\begin{array}{ll}
\partial_{1} \lambda^{2} \frac{\lambda^{4}-\lambda^{3}}{\lambda^{1}-\lambda^{2}}=\partial_{1} \lambda^{3} \frac{\lambda^{4}-\lambda^{2}}{\lambda^{1}-\lambda^{3}}, & \partial_{1} \lambda^{1} \frac{\lambda^{2}-\lambda^{4}}{\lambda^{3}-\lambda^{1}}=\partial_{1} \lambda^{4} \frac{\lambda^{2}-\lambda^{1}}{\lambda^{3}-\lambda^{4}}, \\
\partial_{2} \lambda^{1} \frac{\lambda^{4}-\lambda^{3}}{\lambda^{2}-\lambda^{1}}=\partial_{2} \lambda^{3} \frac{\lambda^{4}-\lambda^{1}}{\lambda^{2}-\lambda^{3}}, & \partial_{2} \lambda^{2} \frac{\lambda^{1}-\lambda^{4}}{\lambda^{3}-\lambda^{2}}=\partial_{2} \lambda^{4} \frac{\lambda^{1}-\lambda^{2}}{\lambda^{3}-\lambda^{4}} \\
\partial_{3} \lambda^{1} \frac{\lambda^{4}-\lambda^{2}}{\lambda^{3}-\lambda^{1}}=\partial_{3} \lambda^{2} \frac{\lambda^{4}-\lambda^{1}}{\lambda^{3}-\lambda^{2}}, & \partial_{3} \lambda^{3} \frac{\lambda^{1}-\lambda^{4}}{\lambda^{2}-\lambda^{3}}=\partial_{3} \lambda^{4} \frac{\lambda^{1}-\lambda^{3}}{\lambda^{2}-\lambda^{4}} .
\end{array}
$$

Немедленным следствием (19) является постоянство двойного отношения

$$
\frac{\left(\lambda^{1}-\lambda^{2}\right)\left(\lambda^{3}-\lambda^{4}\right)}{\left(\lambda^{1}-\lambda^{4}\right)\left(\lambda^{3}-\lambda^{2}\right)}=\mu=\text { const } .
$$

Исключение $\lambda^{4}$ позволяет придать соотношениям (18), (19) следуюший упрошенный вид:

$$
\begin{array}{ll}
\frac{\partial_{1} \partial_{2} \lambda^{1}}{\partial_{1} \lambda^{1} \partial_{2} \lambda^{1}}=\frac{2}{\lambda^{1}-\lambda^{2}}, & \frac{\partial_{1} \partial_{2} \lambda^{2}}{\partial_{1} \lambda^{2} \partial_{2} \lambda^{2}}=\frac{2}{\lambda^{2}-\lambda^{1}}, \\
\frac{\partial_{1} \partial_{3} \lambda^{1}}{\partial_{1} \lambda^{1} \partial_{3} \lambda^{1}}=\frac{2}{\lambda^{1}-\lambda^{3}}, & \frac{\partial_{1} \partial_{3} \lambda^{3}}{\partial_{1} \lambda^{3} \partial_{3} \lambda^{3}}=\frac{2}{\lambda^{3}-\lambda^{1}}, \\
\frac{\partial_{2} \partial_{3} \lambda^{2}}{\partial_{2} \lambda^{2} \partial_{3} \lambda^{2}}=\frac{2}{\lambda^{2}-\lambda^{3}}, & \frac{\partial_{2} \partial_{3} \lambda^{3}}{\partial_{2} \lambda^{3} \partial_{3} \lambda^{3}}=\frac{2}{\lambda^{3}-\lambda^{2}},
\end{array}
$$


а также

$$
\begin{aligned}
\mu\left(\lambda^{1}-\lambda^{3}\right)^{2} \partial_{1} \lambda^{2} & =(\mu-1)\left(\lambda^{1}-\lambda^{2}\right)^{2} \partial_{1} \lambda^{3}, \\
\mu\left(\lambda^{2}-\lambda^{3}\right)^{2} \partial_{2} \lambda^{1} & =\left(\lambda^{1}-\lambda^{2}\right)^{2} \partial_{2} \lambda^{3} \\
(1-\mu)\left(\lambda^{2}-\lambda^{3}\right)^{2} \partial_{3} \lambda^{1} & =\left(\lambda^{1}-\lambda^{3}\right)^{2} \partial_{3} \lambda^{2}
\end{aligned}
$$

так что все соотношения (18), (19) являются следствиями (20), (21). Из (20) вытекает, что

$$
\begin{array}{ll}
\partial_{1} \lambda^{1} \partial_{1} \lambda^{2}=\left(\lambda^{1}-\lambda^{2}\right)^{2} \varphi_{13}, & \partial_{2} \lambda^{1} \partial_{2} \lambda^{2}=\left(\lambda^{1}-\lambda^{2}\right)^{2} \varphi_{23}, \\
\partial_{1} \lambda^{1} \partial_{1} \lambda^{3}=\left(\lambda^{1}-\lambda^{3}\right)^{2} \eta_{12}, & \partial_{3} \lambda^{1} \partial_{3} \lambda^{3}=\left(\lambda^{1}-\lambda^{3}\right)^{2} \eta_{23}, \\
\partial_{2} \lambda^{2} \partial_{2} \lambda^{3}=\left(\lambda^{2}-\lambda^{3}\right)^{2} s_{12}, & \partial_{3} \lambda^{2} \partial_{3} \lambda^{3}=\left(\lambda^{2}-\lambda^{3}\right)^{2} s_{13},
\end{array}
$$

где $\varphi_{i j}\left(R^{i}, R^{j}\right), \eta_{i j}\left(R^{i}, R^{j}\right), s_{i j}\left(R^{i}, R^{j}\right)$ - произвольные функции указанных аргументов. С учетом (21) мы получаем следуюшие связи между $\varphi_{i j}, \eta_{i j}, s_{i j}$ :

$$
\mu \varphi_{13}=(\mu-1) \eta_{12}, \quad \mu \varphi_{23}=s_{12}, \quad(1-\mu) \eta_{23}=s_{13}
$$

откуда

$$
\begin{aligned}
\varphi_{13} & =(\mu-1) \varphi_{1}\left(R^{1}\right), & \varphi_{23} & =\varphi_{2}\left(R^{2}\right), \\
\eta_{12} & =\mu \varphi_{1}\left(R^{1}\right), & \eta_{23} & =\varphi_{3}\left(R^{3}\right), \\
s_{12} & =\mu \varphi_{2}\left(R^{2}\right), & s_{13} & =(1-\mu) \varphi_{3}\left(R^{3}\right),
\end{aligned}
$$

где $\varphi_{i}\left(R^{i}\right)$ - некоторые функции, которые без ограничения общности можно положить равными единице после подходяшей замены инвариантов Римана. Окончательно уравнения (22) перепишутся в виде

$$
\begin{array}{ll}
\partial_{1} \lambda^{1} \partial_{1} \lambda^{2}=(\mu-1)\left(\lambda^{1}-\lambda^{2}\right)^{2}, & \partial_{2} \lambda^{1} \partial_{2} \lambda^{2}=\left(\lambda^{1}-\lambda^{2}\right)^{2} \\
\partial_{1} \lambda^{1} \partial_{1} \lambda^{3}=\mu\left(\lambda^{1}-\lambda^{3}\right)^{2}, & \partial_{3} \lambda^{1} \partial_{3} \lambda^{3}=\left(\lambda^{1}-\lambda^{3}\right)^{2}, \\
\partial_{2} \lambda^{2} \partial_{2} \lambda^{3}=\mu\left(\lambda^{2}-\lambda^{3}\right)^{2}, & \partial_{3} \lambda^{2} \partial_{3} \lambda^{3}=(1-\mu)\left(\lambda^{2}-\lambda^{3}\right)^{2} .
\end{array}
$$

Покажем, что уравнения (23) эквивалентны специальной квадратичной редукции $(2+1)$-мерной системы трех волн. Для этого введем дифференциальные 1-формы

$$
\begin{aligned}
\omega^{1} & =\sqrt{\frac{\mu}{\mu-1}} \frac{\lambda^{1}-\lambda^{3}}{\left(\lambda^{1}-\lambda^{2}\right)\left(\lambda^{2}-\lambda^{3}\right)} d \lambda^{2}-\sqrt{\frac{\mu-1}{\mu}} \frac{\lambda^{1}-\lambda^{2}}{\left(\lambda^{1}-\lambda^{3}\right)\left(\lambda^{2}-\lambda^{3}\right)} d \lambda^{3}, \\
\omega^{2} & =\sqrt{\mu} \frac{\lambda^{2}-\lambda^{3}}{\left(\lambda^{1}-\lambda^{2}\right)\left(\lambda^{1}-\lambda^{3}\right)} d \lambda^{1}-\frac{1}{\sqrt{\mu}} \frac{\lambda^{1}-\lambda^{2}}{\left(\lambda^{1}-\lambda^{3}\right)\left(\lambda^{2}-\lambda^{3}\right)} d \lambda^{3}, \\
\omega^{3} & =-\sqrt{\mu-1} \frac{\lambda^{2}-\lambda^{3}}{\left(\lambda^{1}-\lambda^{2}\right)\left(\lambda^{1}-\lambda^{3}\right)} d \lambda^{1}-\frac{1}{\sqrt{\mu-1}} \frac{\lambda^{1}-\lambda^{3}}{\left(\lambda^{1}-\lambda^{2}\right)\left(\lambda^{2}-\lambda^{3}\right)} d \lambda^{2} .
\end{aligned}
$$

Формы $\omega^{i}$ выбраны с таким расчетом, чтобы уравнения (21) записывались в виде

$$
\omega^{1} \wedge d R^{2} \wedge d R^{3}=0, \quad \omega^{2} \wedge d R^{1} \wedge d R^{3}=0, \quad \omega^{3} \wedge d R^{1} \wedge d R^{2}=0,
$$


причем сами формы $\omega^{i}$ удовлетворяли структурным уравнениям группы Ли $S O(2,1)$ :

$$
d \omega^{1}=\omega^{2} \wedge \omega^{3}, \quad d \omega^{2}=\omega^{3} \wedge \omega^{1}, \quad d \omega^{3}=\omega^{2} \wedge \omega^{1} .
$$

Введем “коэффициенты врашения” $\beta_{i j}$ по формулам

$$
\omega^{1}=\beta_{32} d R^{2}-\beta_{23} d R^{3}, \quad \omega^{2}=\beta_{13} d R^{3}-\beta_{31} d R^{1}, \quad \omega^{3}=\beta_{12} d R^{2}-\beta_{21} d R^{1},
$$

которые являются автоматическим следствием (25). Коэффициенты врашения $\beta_{i j}$ удовлетворяют нелинейной системе уравнений, которая хорошо известна в теории 3 -ортогональных систем координат. Эта система получается подстановкой (27) в структурные уравнения (26):

$$
\begin{array}{ll}
\partial_{1} \beta_{23}=-\beta_{21} \beta_{13}, & \partial_{1} \beta_{32}=-\beta_{31} \beta_{12}, \\
\partial_{2} \beta_{13}=-\beta_{12} \beta_{23}, & \partial_{2} \beta_{31}=-\beta_{32} \beta_{21}, \\
\partial_{3} \beta_{12}=\beta_{13} \beta_{32}, & \partial_{3} \beta_{21}=\beta_{23} \beta_{31},
\end{array}
$$

а также

$$
\begin{aligned}
& \partial_{1} \beta_{12}+\partial_{2} \beta_{21}+\beta_{31} \beta_{32}=0 \\
& \partial_{1} \beta_{13}+\partial_{3} \beta_{31}-\beta_{21} \beta_{23}=0 \\
& \partial_{2} \beta_{23}+\partial_{3} \beta_{32}-\beta_{12} \beta_{13}=0
\end{aligned}
$$

Переход от $\lambda^{i}$ к $\beta_{i j}$ является дифференциальной подстановкой первого порядка. Явные выражения для $\beta_{i j}$ получаются сравнением (27) и (24):

$$
\begin{aligned}
& \beta_{32}=\sqrt{\frac{\mu}{\mu-1}} \frac{\lambda^{1}-\lambda^{3}}{\left(\lambda^{1}-\lambda^{2}\right)\left(\lambda^{2}-\lambda^{3}\right)} \partial_{2} \lambda^{2}-\sqrt{\frac{\mu-1}{\mu}} \frac{\lambda^{1}-\lambda^{2}}{\left(\lambda^{1}-\lambda^{3}\right)\left(\lambda^{2}-\lambda^{3}\right)} \partial_{2} \lambda^{3}, \\
& \beta_{23}=-\sqrt{\frac{\mu}{\mu-1}} \frac{\lambda^{1}-\lambda^{3}}{\left(\lambda^{1}-\lambda^{2}\right)\left(\lambda^{2}-\lambda^{3}\right)} \partial_{3} \lambda^{2}+\sqrt{\frac{\mu-1}{\mu}} \frac{\lambda^{1}-\lambda^{2}}{\left(\lambda^{1}-\lambda^{3}\right)\left(\lambda^{2}-\lambda^{3}\right)} \partial_{3} \lambda^{3}, \\
& \beta_{13}=\sqrt{\mu} \frac{\lambda^{2}-\lambda^{3}}{\left(\lambda^{1}-\lambda^{2}\right)\left(\lambda^{1}-\lambda^{3}\right)} \partial_{3} \lambda^{1}-\frac{1}{\sqrt{\mu}} \frac{\lambda^{1}-\lambda^{2}}{\left(\lambda^{1}-\lambda^{3}\right)\left(\lambda^{2}-\lambda^{3}\right)} \partial_{3} \lambda^{3} \\
& \beta_{31}=-\sqrt{\mu} \frac{\lambda^{2}-\lambda^{3}}{\left(\lambda^{1}-\lambda^{2}\right)\left(\lambda^{1}-\lambda^{3}\right)} \partial_{1} \lambda^{1}+\frac{1}{\sqrt{\mu}} \frac{\lambda^{1}-\lambda^{2}}{\left(\lambda^{1}-\lambda^{3}\right)\left(\lambda^{2}-\lambda^{3}\right)} \partial_{1} \lambda^{3}, \\
& \beta_{12}=-\sqrt{\mu-1} \frac{\lambda^{2}-\lambda^{3}}{\left(\lambda^{1}-\lambda^{2}\right)\left(\lambda^{1}-\lambda^{3}\right)} \partial_{2} \lambda^{1}-\frac{1}{\sqrt{\mu-1}} \frac{\lambda^{1}-\lambda^{3}}{\left(\lambda^{1}-\lambda^{2}\right)\left(\lambda^{2}-\lambda^{3}\right)} \partial_{2} \lambda^{2}, \\
& \beta_{21}=\sqrt{\mu-1} \frac{\lambda^{2}-\lambda^{3}}{\left(\lambda^{1}-\lambda^{2}\right)\left(\lambda^{1}-\lambda^{3}\right)} \partial_{1} \lambda^{1}+\frac{1}{\sqrt{\mu-1}} \frac{\lambda^{1}-\lambda^{3}}{\left(\lambda^{1}-\lambda^{2}\right)\left(\lambda^{2}-\lambda^{3}\right)} \partial_{1} \lambda^{2} .
\end{aligned}
$$

Уравнения (23) приводят, как несложно проверить, к следуюшей квадратичной редукции на коэффициенты $\beta_{i j}$ :

$$
\frac{\beta_{32}^{2}}{\mu}-\beta_{12}^{2}=-4, \quad \frac{\beta_{23}^{2}}{\mu-1}-\beta_{13}^{2}=4, \quad \frac{\beta_{21}^{2}}{\mu-1}-\frac{\beta_{31}^{2}}{\mu}=4 .
$$

4 Теоретическая и математическая физика, т. 110, № 1, 1997 г. 
Заметим, что уравнения (28) представляют собой $(2+1)$-мерную систему трех волн, интегрируемую при помощи линейной задачи

$$
\begin{array}{ll}
\partial_{2} H^{1}=-\beta_{21} H^{2}, & \partial_{3} H^{1}=-\beta_{31} H^{3}, \\
\partial_{1} H^{2}=-\beta_{12} H^{1}, & \partial_{3} H^{2}=-\beta_{32} H^{3}, \\
\partial_{1} H^{3}=\beta_{13} H^{1}, & \partial_{2} H^{3}=\beta_{23} H^{2} .
\end{array}
$$

Чтобы удовлетворить редукции (30), положим

$$
\begin{array}{ll}
\beta_{32}=2 \sqrt{\mu} \operatorname{sh} u, & \beta_{12}=2 \operatorname{ch} u, \\
\beta_{23}=2 \sqrt{\mu-1} \operatorname{ch} v, & \beta_{13}=2 \operatorname{sh} v, \\
\beta_{21}=2 \sqrt{\mu-1} \operatorname{ch} w, & \beta_{31}=2 \sqrt{\mu} \operatorname{sh} w .
\end{array}
$$

В результате такой подстановки уравнения (28) перепишутся в виде

$$
\begin{aligned}
\partial_{1} u & =-2 \operatorname{sh} w, & \partial_{3} u & =2 \sqrt{\mu} \operatorname{sh} v, \\
\partial_{1} v & =-2 \operatorname{ch} w, & \partial_{2} v & =-2 \sqrt{\mu-1} \operatorname{ch} u, \\
\partial_{2} w & =-2 \sqrt{\mu-1} \operatorname{sh} u, & \partial_{3} w & =2 \sqrt{\mu} \operatorname{ch} v
\end{aligned}
$$

(заметим, что уравнения (29) при этом оказываются выполненными тождественно). После подходящей нормировки инвариантов Римана уравнения (31) примут простой вид

$$
\begin{aligned}
\partial_{1} u & =\operatorname{sh} w, & \partial_{3} u & =\operatorname{sh} v, \\
\partial_{1} v & =\operatorname{ch} w, & \partial_{2} v & =\operatorname{ch} u, \\
\partial_{2} w & =\operatorname{sh} u, & \partial_{3} w & =\operatorname{ch} v .
\end{aligned}
$$

Выражая отсюда, например, $v$ и $w$ в виде

$$
v=\operatorname{arcsh} \partial_{3} u, \quad w=\operatorname{arcsh} \partial_{1} u,
$$

мы можем переписать полученную систему в виде трех попарно коммутируюших уравнений Монжа-Ампера

$$
\begin{aligned}
& \partial_{1} \partial_{2} u=\operatorname{sh} u \sqrt{1+\left(\partial_{1} u\right)^{2}}, \\
& \partial_{3} \partial_{2} u=\operatorname{ch} u \sqrt{1+\left(\partial_{3} u\right)^{2}}, \\
& \partial_{1} \partial_{3} u=\sqrt{1+\left(\partial_{1} u\right)^{2}} \sqrt{1+\left(\partial_{3} u\right)^{2}}
\end{aligned}
$$

(общая задача классификации коммутирующих уравнений Монжа-Ампера, по-видимому, ранее не рассматривалась). Представляет интерес дальнейшее изучение конечных семейств систем гидродинамического типа порядка $n \times n$ в инвариантах Римана, замкнутых относительно всей совокупности преобразований Лапласа $S_{i j}$. При этом естественно рассмотреть семейства, для которых $S_{i j}^{k}=\mathrm{id}$ при некотором $k \geq 2$. Как показывают разобранные выше примеры, эта задача нетривиальна уже для $(n=2, k=2)$ и $(n=3, k=2)$. По-видимому, уравнения на характеристические скорости соответствующих систем должны сводиться к подходящим редукциям $(2+1)$-мерной системы

$$
\partial_{k} \beta_{i j}=\beta_{i k} \beta_{k j}
$$

Автор благодарит участников семинара "Геометрия и математическая физика" под руководством С. П. Новикова за полезные обсуждения.

Работа выполнена при частичной финансовой поддержке РФФИ (гранты 96-01-00166, 96-06-80104a) и INTAS. 


\section{Список литературы}

[1] С. П. Царев. Изв. АН СССР, сер. матем. 1990. Т. 54. № 5. С. 1048-1068.

[2] G. Darboux. Lecons sur la theorie generale des surfaces. Part 4. Paris: Gautier-Villars.

[3] A. B. Shabat, R. A. Yamilov. To transformation theory of two-dimensional integrable systems. (To appear in Phys. Lett. A.)

[4] R. I. Yamilov. Classification of Toda type scalar lattices. Proc. 8th Int. Workshop on Nonlinear Evolution Equations and Dynamical Systems. World Sci. Publishing, 1993. P. 423-431.

[5] А. Б. Шабат, Р. И. Ямилов. Алгебра и анализ. 1990. 2Вып. 2. С. 183-208.

[6] А.П. Веселов, С.П. Новиков. УМН. 1995. Вып. 6. С. 171-172.

[7] Т. Л. Козьмина. ДАН. 1947. Т. 55. № 3. С. 187-189.

[8] Р. В. Смирнов. ДАН. 1950. Т. 71. № 3. С. 437-439.

[9] Т. А. Шульман. ДАН. 1952. Т. 85. № 3. С. 501-504.

[10] M. A. Akivis, V. V. Goldberg. Rend. Sem. Mat. Messina, ser II. 1991. V. 1. P. 9-29.

[11] N. Kamran, K. Tenenblat. Duke Math. J. 1996. V. 84. № 1. P. 237-266.

[12] C. Athorne. Phys. Lett. A. 1995. V. 206. P. 162-166.

Поступила в редакцию 28.III.1996 г.

\section{E. V. Ferapontov \\ LAPLACE TRANSFORMATIONS OF HYDRODYNAMIC TYPE SYSTEMS IN RIEMANN INVARIANTS}

The conserved densities of hydrodynamic type system in Riemann invariants satisfy a system of linear second order partial differential equations. For linear systems of this type Darboux introduced Laplace transformations, generalizing the classical transformations of a scalar equation of the second order. It is demonstrated, that Laplace transformations can be pulled back to the transformations of the corresponding hydrodynamic type systems. We discuss finite families of hydrodynamic type systems, that are closed under all Laplace transformations. For $3 \times 3$ systems in Riemann invariants we propose a complete discription of closed quadruples. They turn to be related to a special quadratic reduction of the $(2+1)$-dimensional 3 -wave system. 